\title{
Desmoplastic fibroma of the distal tibia: A case report of a minimally invasive histological diagnosis
}

\author{
GABRIELE LEVRINI and PIERPAOLO PATTACINI \\ Department of Diagnostic Imaging, Arcispedale Santa Maria Nuova-IRCCS, I-42100 Reggio Emilia, Italy
}

Received February 9, 2016; Accepted August 8, 2016

DOI: $10.3892 / \operatorname{mco} .2016 .1022$

\begin{abstract}
Desmoplastic fibroma (DF) is a benign, rare fibroblastic intraosseous neoplasm histologically resembling a desmoid soft tissue tumor. Although classified as benign, DF frequently exhibits an aggressive behavior, has a moderate-to-high recurrence rate, and often causes pathological fractures and extensive bone destruction. This case report presents an incidentally detected DF of the tibia, which was diagnosed using a minimally invasive approach. A 36-year-old African female patient was referred to the Department of Diagnostic Imaging of Arcispedale Santa Maria Nuova-IRCCS (Reggio Emilia, Italy), to be examined by a computed tomography scan on an outpatient basis, after an x-ray examination of the tibia, which was performed after an injury to exclude the presence of a fracture, revealed a hyperlucency of unknown origin. The aim of this study was to discuss the clinical, histological, immunohistochemical and radiographic characteristics of this rare neoplasm, with a focus on image-guided bone biopsy.
\end{abstract}

\section{Introduction}

DF of the bone is an extremely rare, lytic, locally aggressive but non-metastatic primary bone tumor. Histologically, DF consists of wavy fibroblasts and abundant collagenous tissue, bears a close resemblance to a desmoid tumor of the soft tissues and exhibits a characteristic production of collagen fibers (1). DF may involve any bone, but is most often found in the mandible (22\%) and less frequently in the femur (15\%), pelvic bones $(13 \%)$, radius $(12 \%)$ and tibia $(9 \%)(2,3)$.

The incidence of DF is estimated to be $0.06 \%$ among all bone tumors and $0.3 \%$ among benign bone tumors $(1,3-5)$. DF of the bone most often occurs during the first three decades of

Correspondence to: $\mathrm{Dr}$ Gabriele Levrini, Department of Diagnostic Imaging, Arcispedale Santa Maria Nuova-IRCCS, 80 Viale Risorgimento, I-42100 Reggio Emilia, Italy

E-mail: gabriele.levrini@asmn.re.it; levrinig@gmail.com

Key words: computed tomography-guided biopsy, desmoplastic fibroma, bone life and exhibits no gender predilection (6). The most common symptoms include pain and swelling, and $12 \%$ of the patients present with pathological fractures. However, a number of patients may be asymptomatic; thus, the tumor is often incidentally identified (7).

On radiographs, the lesion is lytic, occasionally with poorly defined borders, while on computed tomography (CT) it is mildly hyperdense, with thin sclerotic margins, with or without destruction of the contiguous cortex, and exhibits low signal intensity on T2-weighted magnetic resonance imaging (MRI). The diagnosis of DF is predominantly based on pathological examination. The recurrence rates in patients treated with and without resection are 17 and 55-72\%, respectively (7).

This disease was first identified by Jaffe in 1958 (8). To date, 200 cases of desmoplastic fibroma of the bone have been reported in the literature; of these, 13 have been reported in the femur (9-20), with only 2 in the proximal femur $(9,14)$ and the remaining in the distal femur (10-13,15-18,20,21).

Image-guided percutaneous biopsy is becoming an increasingly accepted modality for initial diagnosis in the majority of musculoskeletal tumors. However, despite its well-documented specificity and sensitivity $(22,23)$, there has been continued reluctance on the part of orthopedic oncologists to accept this technique as the modality of choice for initial biopsy in primary bone tumors. Detractors of percutaneous needle biopsy suggest that sufficient material cannot be obtained to ensure an accurate diagnosis, particularly in patients with sclerotic bone lesions (24). Historically, all biopsies to date were performed via an open surgical biopsy, which remains the gold standard for a proportion of physicians. The reported accuracy of an open surgical biopsy, whether incisional or excisional, is $98 \%(23,25)$, whereas needle biopsy under CT guidance has a reported accuracy estimated between 78 and $98.4 \%$ (26).

The needle pathway is determined on the basis of the expected definitive treatment, in order to avoid intercompartmental contamination, minimize the amount of biopsy tract to be removed at the time of definitive surgery in case of malignancy, and avoid neurovascular structures. The choice of CT vs. fluoroscopy is generally determined on the basis of three factors, namely size, location and attenuation (27).

To the best of our knowledge, this study presents the only published case of minimally invasive diagnosis of DF of the bone. Written informed consent was obtained from the patient for the publication of the case details. 


\section{Case report}

In August 2015, a 36-year-old African female patient presented to the Emergency Room of Arcispedale Santa Maria Nuova-IRCCS (Reggio Emilia, Italy), which is a medium-sized general hospital, complaining of pain in the rib cage, knees and lower thighs following a mild trauma she had suffered a few days earlier after a fall, for which she had not been hospitalized. There were no evident abnormalities on physical examination, with the exception of pain on percussion. Radiography showed a well-defined, eccentric, low-density lesion in the distal metaphysis of the left tibia (Fig. 1), adherent to the external cortex. The contiguous cortex was apparently not eroded, but minimally thinned due to compression by the expanding tumor. The margins were intact and sclerotic. The initial diagnosis on admission, based on radiological evidence, was bone fibroma. The patient was advised on an outpatient basis to undergo a magnetic resonance imaging (MRI) scan and a subsequent orthopedic evaluation.

Due to the X-ray and bone MRI results, the orthopedic surgeon suggested an image-guided bone biopsy.

After a CT scan confirmed the location and imaging characteristics of the lesion (solid, well-circumscribed, no cortex erosions), local anesthesia with lidocaine was administered, a 17-gauge bone biopsy needle (Osteo-Site Ratchet; Cook Medical, Limerick, Ireland) was inserted, passing through the anterolateral face of the skin overlying the tibia, the tibialis anterior muscle and, finally, the cortex, into the core of the lesion (Fig. 2) and a single specimen was extracted. Since no peri-procedural complications occurred, the patient was discharged after a few hours in good health and without the need for oral pain medication, with the recommendation to refer to her general practitioner in case of any discomfort. Histological examination of the bone specimen revealed focal peripheral proliferation of spindle-shaped cells resembling fibroblasts, showing strong reactivity for anti-vimentin antibody; the appearance was in accordance with a fibroblastic desmoid tumor of the bone.

\section{Discussion}

Percutaneous needle bone biopsy is a safe and accurate method $(22,25,27-29)$ for obtaining a tissue diagnosis. In general, better results are obtained with lesions of the extremities or pelvis compared with those in the spine (30). Percutaneous needle biopsies have a very low complication rate $(1.1 \%)$, whereas open biopsy has a complication rate of $\leq 16 \%(31)$.

A serious complication of percutaneous biopsy is the risk of seeding malignant cells along the needle track, particularly if the lesion is a sarcoma, which would necessitate resection of the needle track en bloc with the tumor in limb-sparing reconstructive surgery (32). Thus, selecting the appropriate needle path is critical for limb-salvaging procedures. Fine-needle aspiration (FNA) may differentiate between a metastasis and a benign lesion; however, core biopsy is superior to FNA in determining cell type and tumor grade, which is necessary for the diagnosis of primary bone tumor. In our experience, of the various options that are available for bone biopsy (33), the Osteo-Site Ratchet (Cook Medical) 13-gauge coaxial bone

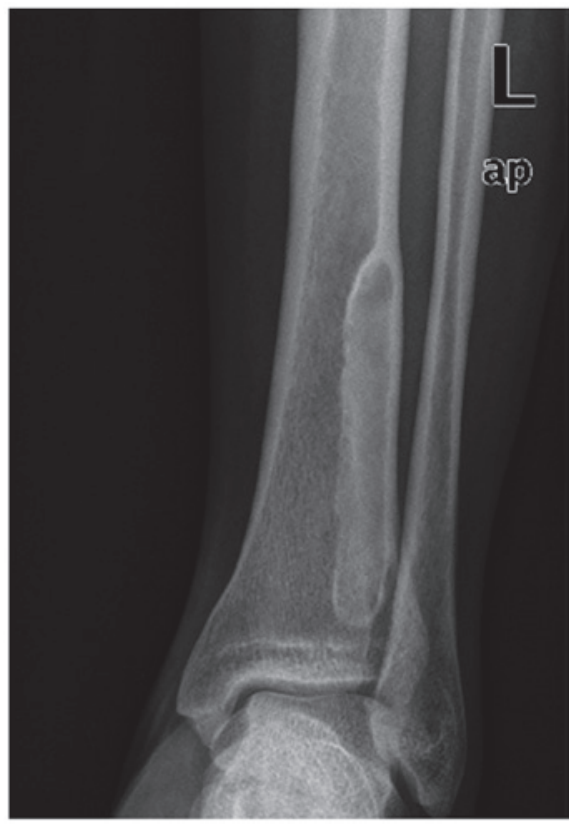

Figure 1. X-ray of the left tibia showing a well-defined, eccentric, low-density lesion in the distal metaphysis.

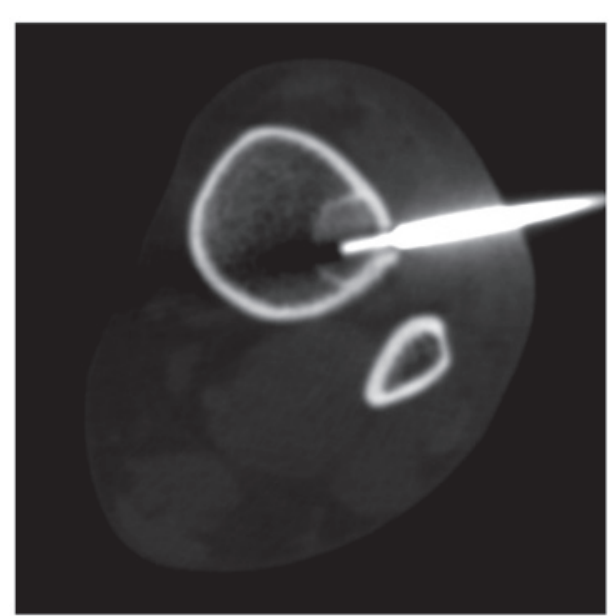

Figure 2. Computed tomography axial image (bone window setting) showing a solid, mildly hyperdense, well-circumscribed, eccentric lesion in the distal metaphysis of the left tibia, not eroding the cortex (note the distal part of biopsy needle inside the lesion).

biopsy system is effective in obtaining tissue from sclerotic lesions where normal bone cortex needs to be traversed. In general, all bone biopsies are performed under local anesthesia; however, conscious sedation may be required in children or uncooperative patients. An adequate biopsy sample is considered to be that providing adequate abnormal tissue for the pathologist to reliably make a diagnosis. We have found that, despite the low incidence of DF, a single long core of abnormal tissue obtained with a 13-gauge needle is sufficient.

In conclusion, this study reported the case of a 36-year-old African female patient with a DF of the bone incidentally detected by an X-ray and diagnosed by means of percutaneous CT-guided bone biopsy. This case differs from other published studies due to the minimally invasive, preoperative, image-guided histological diagnosis. This type of diagnosis 
is commonly made based on a resection sample obtained through incisional biopsy under general anesthesia. The results of image-guided biopsy in terms of cost-to-benefit ratio are similar to those of open biopsy, with reduced comparable morbidity.

\section{References}

1. Böhm P, Kröber S, Greschniok A, Laniado M and Kaiserling E: Desmoplastic fibroma of the bone. A report of two patients, review of the literature, and therapeutic implications. Cancer 78: 1011-1023, 1996

2. Fletcher CDM, Uni KK and Mertens F: Pathology and genetics of tumors of soft tissue and bone. WHO classification of tumors. IARC Press, Lyon, p288, 2002.

3. Said-Al-Naief N, Fernandes R, Louis P, Bell W and Siegal GP: Desmoplastic fibroma of the jaw: A case report and review of literature. Oral Surg Oral Med Oral Pathol Oral Radiol Endod 101: 82-94, 2006.

4. Rabin D, Ang LC, Megyesi J, Lee DH and Duggal N: Desmoplastic fibroma of the cranium: Case report and review of the literature. Neurosurgery 52: 950-954; discussion 954, 2003.

5. Ikeshima A and Utsunomiya T: Case report of intra-osseous fibroma: A study on odontogenic and desmoplastic fibromas with a review of the literature. J Oral Sci 47: 149-157, 2005.

6. Crim JR, Gold RH, Mirra JM, Eckardt JJ and Bassett LW: Desmoplastic fibroma of bone: Radiographic analysis. Radiology 172: 827-832, 1989.

7. Hauben E and Cleton-Jansen AM: Fibrogenic tumours: Desmoplastic fibroma of bone. In: WHO Classification of Tumours of Soft Tissue and Bone. Fletcher CDM, Bridge JA, Hogendoorn P and Mertens F (eds). 4th edition. IARC, Lyon, p298, 2013

8. Jaffe HL: Tumors and tumorous conditions of the bones and joints. In: in The American Journal of the Medical Sciences Lea \& Febiger, Philadelphia, 1958.

9. Lichtman EA and Klein MJ: Case report 302. Desmoplastic fibroma of the proximal end of the left femur. Skeletal Radiol 13 160-163, 1985

10. Wadhwa V, Suh KJ, Yi JH and Chhabra A: Incidental lesion in the femoral metaphysis. Desmoplastic fibroma of the bone. Skeletal Radiol 42: 1739-1740, 1775-1776, 2013.

11. Clayer M and Oakeshott R: Allograft bone in the treatment of desmoplastic fibroma. A case report. Clin Orthop Relat Res 219-224, 1994

12. Rastogi S, Varshney MK, Trikha V, Khan SA and Mittal R: Desmoplastic fibroma: A report of three cases at unusual locations. Joint Bone Spine 75: 222-225, 2008

13. Chan KW, Pun WK and Choi CH: Desmoplastic fibroma of bone. Pathology 19: 201-203, 1987

14. Grube-Pagola P and Valle-Landa JC: Desmoplastic fibroma of the femur. Radiologia 55: 359-361, 2013 (In Spanish).

15. Doya $\mathrm{H}$ and Yaoita M: Case of desmoplastic fibroma in the femur. Seikei Geka 17: 754-756, 1966 (In Japanese).

16. Matsumori S, Watabe E, Toyoshima Y and Yagi Y: Primary desmoplastic fibroma of the femur. Seikei Geka 23: 275-281, 1972 (In Japanese).

17. Bertoni F, Calderoni $\mathrm{P}$, Bacchini $\mathrm{P}$ and Campanacci $\mathrm{M}$ : Desmoplastic fibroma of bone. A report of six cases. J Bone Joint Surg Br 66: 265-268, 1984
18. Petrovichev NN, Filippova NA, Annamukhammedov A and Karapetian RM: Case of desmoplastic fibroma of the femur. Arkh Patol 47: 55-58, 1985 (In Russian).

19. Nielsen GP, O'Connell JX, Dickersin GR and Rosenberg AE: Collagenous fibroma (desmoplastic fibroblastoma): A report of seven cases. Mod Pathol 9: 781-785, 1996.

20. Gao S, Cai Q, Yao W, Wang J, Zhang P and Wang X: Desmoplastic (collagenous) fibroma of the femur: A case report and review of the literature. Oncol Lett 6: 1285-1288, 2013.

21. Yokouchi M, Ueno Y, Nagano S, Shimada H, Nakamura S, Setoguchi T, Kawamura I, Ishidou Y and Komiya S: Extended curettage and heat ablation for desmoplastic fibroma of the distal femur with a 12-year follow-up period: A case report. Oncol Lett 8: 1103-1106, 2014.

22. Fraser-Hill MA and Renfrew DL: Percutaneous needle biopsy of musculoskeletal lesions. 1. Effective accuracy and diagnostic utility. AJR Am J Roentgenol 158: 809-812, 1992.

23. Dupuy DE, Rosenberg AE, Punyaratabandu T, Han MH and Mankin HJ: Accuracy of CT-guided needle biopsy of musculoskeletal neoplasms. AJR Am J Roentgenol 171: 759-762, 1998.

24. Mankin HJ, Mankin CJ and Simon MA: The hazards of biopsy, revisited. Members of the musculoskeletal tumor society. J Bone Joint Surg Am 78: 656-663, 1996.

25. Altuntas AO, Slavin J, Smith PJ, Schlict SM, Powell GJ, Ngan S, Toner $\mathrm{G}$ and Choong PF: Accuracy of computed tomography guided core needle biopsy of musculoskeletal tumours. ANZ J Surg 75: 187-191, 2005.

26. Rimondi E, Rossi G, Bartalena T, Ciminari R, Alberghini A Ruggieri P, Errani C, Angelini A, Calabrò T, Abati CN, et al: Percutaneous CT-guide biopsy of the musculoskeletal system: Results of 2027 cases. Eur J Radiol 77: 34-42, 2011.

27. Jelinek JS, Murphey MD, Welker JA, Henshaw RM, Kransdorf MJ, Shmookler BM and Malawer MM: Diagnosis of primary bone tumors with image-guided percutaneous biopsy: Experience with 110 tumors. Radiology 223: 731-737, 2002.

28. Leffler SG and Chew FS: CT-guided percutaneous biopsy of sclerotic bone lesions: Diagnostic yield and accuracy. AJR 172: 1389-1392, 1999.

29. Ashford RU, McCarthy SW, Scolyer RA, Bonar SF, Karim RZ and Stalley PD: Surgical biopsy with intraoperative frozen section. An accurate and cost-effective method for diagnosis of musculoskeletal sarcomas. J Bone Joint Surg Br 88: 1207-1211, 2006.

30. Hau A, Kim I, Kattapuram S, Hornicek FJ, Rosenberg AE, Gebhardt MC and Mankin HJ: Accuracy of CT-guided biopsies in 359 patients with musculoskeletal lesions. Skeletal Radiol 31: 349-353, 2002

31. Welker JA, Henshaw RM, Jelinek J, Shmookler BM and Malawer MM: The percutaneous needle biopsy is safe and recommended in the diagnosis of musculoskeletal masses. Cancer 89: 2677-2686, 2000

32. Anderson MW, Temple HT, Dussault RG and Kaplan PA: Compartmental anatomy: Relevance to staging and biopsy of musculoskeletal tumors. AJR Am J Roentgenol 173: 1663-1671, 1999.

33. Roberts CC, Morrison WB, Leslie KO, Carrino JA, Lozevski JL and Liu PT: Assessment of bone biopsy needles for sample size, specimen quality and ease of use. Skeletal Radiol 34: 329-335, 2005. 